\title{
Segmentation and Classification Method in IVOCT Images
}

\author{
Zhou Ping ${ }^{1, a^{*}}$, Zhu Tongjing ${ }^{1, b}$, Li Zhiyong ${ }^{1, c}$
}

${ }^{1}$ School of Biological Science \& Medical Engineering, Southeast university, Nanjing, 210096, china;

acapzhou@163.com, bzhutongjing@163.com, ${ }^{\mathrm{c}} 101011308 @$ seu.edu.cn

Keywords: intravascular; optical coherence tomography; lumen detection; plaque classification

\begin{abstract}
Cardiovascular disease (CVD) is a fatal disease of the heart or blood vessels. Intravascular optical coherence tomography (IVOCT) as a newly emerging optical-based technology can provide real-time, high-resolution, and three dimensional images with micrometer resolution. In this paper, an automatic lumen detection method composed of OTSU threshold and active contour model, was investigated to improve the robustness and accuracy. The proposed method is compared with manual lumen detection (MLD), and then average distance and max distance results are obtained. For the given datasets, the average distance and max distance is $0.020 \mathrm{~mm}$ and $0.088 \mathrm{~mm}$ respectively. Furthermore, an automatic plaque segmentation and classification is proposed to use Hidden Markov Models(HMM), GLCM and Random Forests algorithm. From the color-code plaque classification results, the approach proposed is available. In conclusion, this method can deal with IVOCT image with high robustness and accuracy.
\end{abstract}

\section{Introduction}

Currently, cardiovascular disease (CVD) is fatal disease of the heart or blood vessels. Intravascular Optical coherence tomography (IVOCT) is a newly promising imaging technology, which has greater advantages than other technologies in resolution. Traditionally, OCT lumen detection and plaque characterization were performed manually which is time consuming. On the contrary, automated image analysis can (1) reduce the heavily burdens of operators, (2) provide more comprehensive diagnosis, and (3) help reduce interobserver variability ${ }^{[1]}$.

The automated analysis method produced in previous studies consists of two parts: the lumen border detection and the plaque classification. Many research groups have proposed several algorithm for detecting vascular wall contour. Gurmeric et al. proposed that the lumen border can be segmented by using threshold method and Catmull-Rom spline interpolation algorithm ${ }^{[2]}$. Moraes et al. presented lumen segmentation, based on wavelets and mathematical morphology ${ }^{[3]}$. Tsantis et al. used a MRF model and CWT transformation to estimate the shape of the lumen border ${ }^{[4]}$. After the lumen detection, plaque classification can be performed using optical properties or various tissue features. $\mathrm{Xu}$ et al. proposed a single scattering model to extract the backscattering and attenuation coefficients of tissue ${ }^{[5]}$. Ughi et al. selected regions of interest by using the attenuation coefficient estimates and then classified the issues by spatial gray-level dependence matrices (SGLDMs) ${ }^{[6]}$.

In this study, we introduce a systematic approach for the segmentation of the lumen border and the characterization of intravascular plaques (calcium, lipid, fibrous). Firstly, an automatic lumen detection method was investigated to improve the robustness and accuracy. This method is based on OTSU threshold and active contour model. Secondly, identification of the different plaque roughly relies on tissue region detection by HMM method and accurately plaque identification by machine learning techniques.

\section{Method}

\subsection{Lumen Detection.}

An automatic lumen detection method was investigated to improve the robustness and accuracy. Firstly, we roughly detect the lumen by OTSU threshold method. After polar transformation, a 
active contour model is used to correct the contour of the lumen border and to extrapolate the contour in the area of the guide-wire shadow.

Otsu's threshold. Otsu's method is used to automatically perform IVOCT image thresholding to generate a binary image. However, as the signal intensity drops off with the detection depth, the yonder organizations usually miss identification. In order to improve the detection sensitivity, we do OTSU threshold for each line rather than full image. Fig.1(a) shows the test result of the OTSU line by line while Fig.1(b) shows the result of the complete OTSU image. The sensibility of signal testing is increased. The contour within the vascular wall is obtained by transforming the image from polar to Cartesian coordinates.

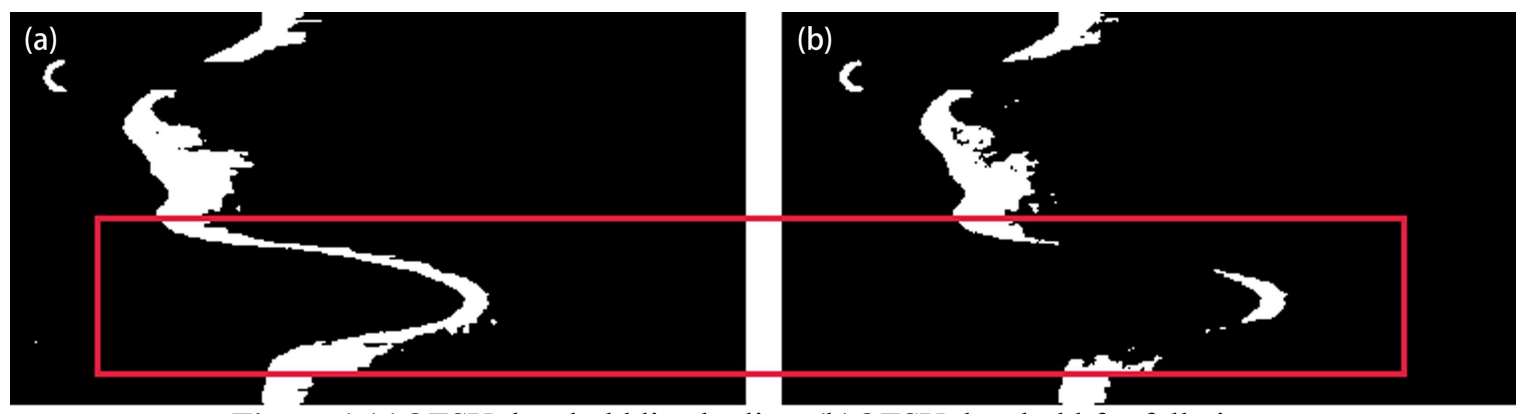

Figure.1 (a)OTSU threshold line by line, (b)OTSU threshold for fully image

Active Contour Model. The contour is deformed under the pressure of internal energy and external energy. While the external energy draws active contour to move towards the edge of an object, the internal energy keep the active contour smooth and connective. When energy diminishes to minimum value, the active contour arrives at the edge of the object. Assumed the closed curve $v(s)$ constitutes a contour, its internal energy is depicted as

$$
E_{\text {int }}(v)=\int_{0}^{1}\left(\alpha\left|v_{s}(s)\right|^{2}+\beta\left|v_{s s}(s)\right|^{2}\right) d s .
$$

Where $\alpha\left|v_{s}(s)\right|^{2} \quad$ is elastic energy, $\beta\left|v_{s s}(s)\right|^{2} \quad$ is curving energy, $\alpha$ and $\beta$ are artificial parameters, $v_{s}(s)$ and $v_{s s}(s)$ represent the first- and second-order derivative of the curve.

The external energy is derived from

$$
E_{\text {ext }}(v)=\int_{0}^{1} P(v(s)) d s=-|\nabla I(v)|^{2} .
$$

The curve total energy is

$$
E_{\text {snake }}=E_{\text {int }}(v)+E_{\text {ext }}(v) \text {. }
$$

The position of the contour as shown in Fig.2(a) is assessed by the external energy and force field. The active contour model is used to make the total energy lowest by iteration, so that the contour matchs the contour shaping excellently, as shown in Fig.2(d).

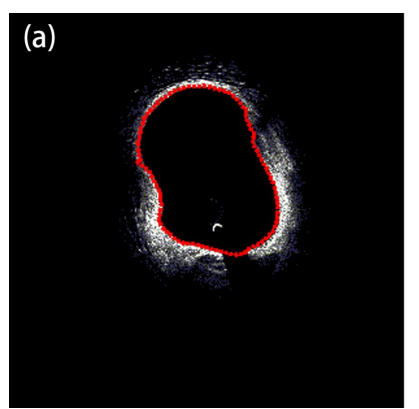

(b)

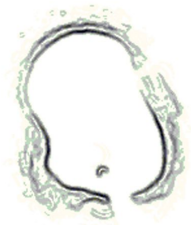

(c)

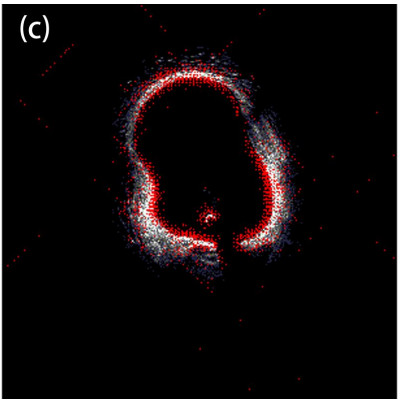

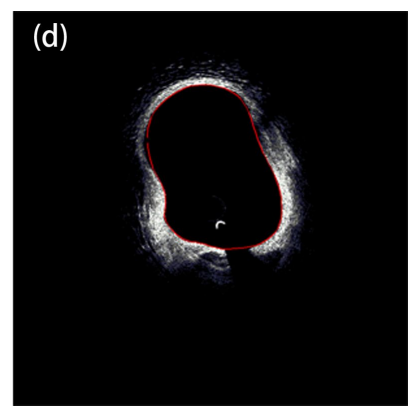

Figure.2 (a)previous contour, (b)external energy, (c)external force field, (d)accuracy detection result

\subsection{Plaque Segmentation and Classification}

An automatic plaque segmentation and classification method are applied by four setps: (1) 
plaque region segmentation by HMM, (2) calcium plaque detection following Zhao Wang's method, (3) gray level co-occurrence matrices(GLCM) texture feature vectors extraction and (4) plaque classification using Random Forests.

Plaque region segmentation. As lumen border is precisely confirmed, it is considered as the internal boundary of tissue area and the profile beyond $2 \mathrm{~mm}$ is regarded as the external boundary, as shown in Fig.3(a). HMM is implemented to get plaque regeion, as shown in Fig. 3(b).
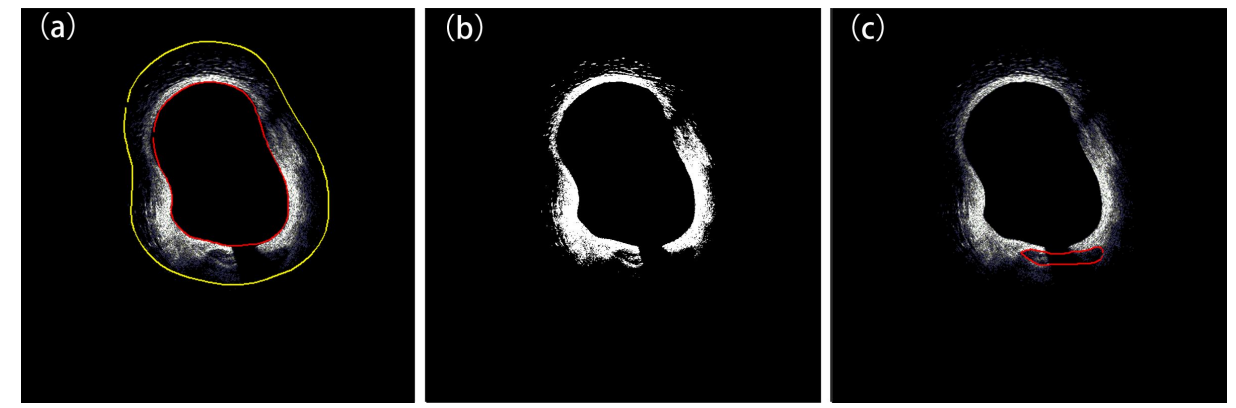

Figure.3 (a)the internal and external boundary of tissue area, (b)the plaque region identified by means of HMM, (c)the calcium detection result

Calcium Plaque Detection. Three plaque types have different characteristics: calcium (CA), that appears as a non-homogeneous region with sharply delineated borders, fibrous tissue (FT), that is describe as a high back-scattering homogeneous area, and lipid tissue (LT), that is shown as a signal poor region. Therefore, the FT and LT can be identified by machine learning techniques. Following the Zhao Wang's method ${ }^{[7]}$, the CA plaque is localized by edge detection and traced using a combined intensity and gradient-based active counter model. The results are shown in Fig.3(c).

Feature Extraction. For each pixel $I(i, j)$ in the tissue region without calcium, a $11 \times 11$ pixels neighborhood and CLCM are used to compute the feature vectors. The GLCM characterizes the texture of an image by calculating how often pairs of pixel with specific distance $(D)$ and orientation $(\theta)$ occur in the image. We create four GLCMs $C_{D, \theta}(i, j)$ with $D=2$ and all values of $\theta$ $(\theta=0,45,90,135 \mathrm{deg})$, and for each GLCM $C_{D, \theta}(i, j)$ the statistics providing information about the texture are obtained. Therefore, 16 GLCM features along with the entropy and mean value of the $11 \times 11$ neighborhood forms a vector of 18 features for each pixel in the plaque region. The textural features of the lipid and fibrous plaques are listed in table 2.

Classification. All the previous feature vectors are used as input to Random Forests(RF) algorithm. $\mathrm{RF}$ is an ensemble learning method for classification, regression and other tasks, that operate by constructing a multitude of decision trees at training time and outputting the class that is the model of the classes. After parameter tuning, we use the specific with 100 trees and 8 variables. Each of feature vectors is classified into two classes: (1) fibrous tissue and (2) lipid tissue. Hence, classification results can be displayed in color-coded as shown in Fig.4(b).
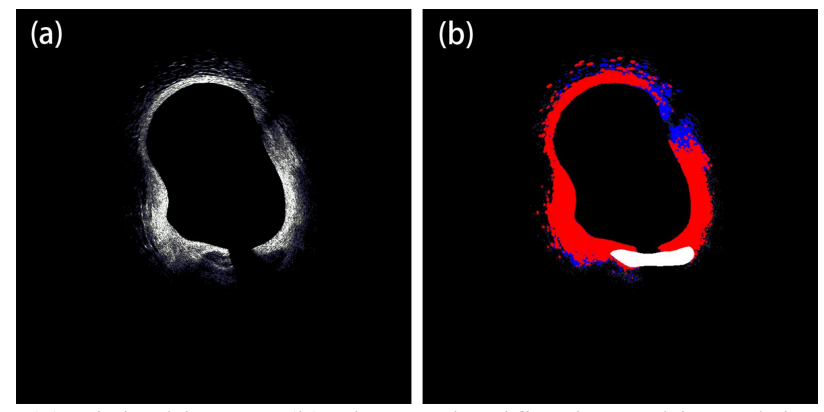

Figure.4 (a)original image, (b) plaque classification: white-calcium plaque, Red-fibrous plaque, blue-lipid plaque

\section{Results}

In the experiments on IVOCT, its imaging is collected by means of the FD-OCT CRX7 system. 
Each dataset contains about 200 frames of images. In order to check algorithm precision, three datasets are tested. Firstly, through a manual outlining test, the located outlining of artery walls are regarded as a standard. Then, the averages and maximums within the results of the automatic and manual tests are calculated by the method of this paper.

Table 1. The Distance between Automatic Detection Result and Manual Detection Result

\begin{tabular}{ccc}
\hline & Average distance $[\mathrm{mm}]$ & Maximum distance $[\mathrm{mm}]$ \\
\hline DataSet1 & 0.025 & 0.087 \\
DataSet2 & 0.011 & 0.095 \\
DataSet3 & 0.024 & 0.081 \\
\hline
\end{tabular}

For the given datasets, the proposed method was implemented to calculate the texture features of lipid and fibrous plaques. In table 2, we exhibit the mean features values under the parameter of 0 and $90 \mathrm{deg}$. Clearly, there's a significant difference between lipid and fibrous, that can be a great benefit to classification.

Table 2. Measured Values of Textural Features of the lipid and Fibrous Plaques

\begin{tabular}{lccccc}
\hline & Contrast & Energy & Homogeneity & Correlation & Mean \\
\hline lipid $(0 \mathrm{deg})$ & 186.3882 & 0.0133 & 0.3058 & 0.0031 & 86.8564 \\
lipid $(90 \mathrm{deg})$ & 208.1347 & 0.0127 & 0.2346 & 0.0022 & 88.7692 \\
Fibrous $(0 \mathrm{deg})$ & 1672.7607 & 0.0018 & 0.1135 & 0.0002 & 182.7505 \\
Fibrous $(90 \mathrm{deg})$ & 2064.7399 & 0.0028 & 0.1665 & 0.0001 & 184.4397 \\
\hline
\end{tabular}

\section{Conclusions}

In this study, the proposed lumen detection approach is compared with manual lumen detection (MLD), and average distance and max distance results are obtained. For the given datasets, the average distance and max distance is $0.020 \mathrm{~mm}$ and $0.088 \mathrm{~mm}$. It shows that the method of this paper can precisely obtain the tested images of lumen border. Furthermore, there is a significant different between lipid and fibrous in some statistics such as contrast, energy and correlation. From the color-code plaque classification results, the approach proposed is available. In conclusion, this method can automatic analyze IVOCT image with high robustness and accuracy.

\section{References}

[1] Ligthart J, Bruining N, van Soest G. The diagnostic value of intracoronary optical coherence tomography, J. Herz, 2011, 36(5): 417-429.

[2] Gurmeric, Serhan, et al. A new 3-D automated computational method to evaluate in-stent neointimal hyperplasia in in-vivo intravascular optical coherence tomography pullbacks. Medical Image Computing and Computer-Assisted Intervention-MICCAI 2009. Springer Berlin Heidelberg, 2009. 776-785.

[3] Moraes M C, Cardenas D A C, Furuie S S. Automatic IOCT lumen segmentation using wavelet and Mathematical Morphology. Computing in Cardiology (CinC), 2012. IEEE, 2012: 545-548.

[4] Tsantis S, Kagadis G C, Katsanos K, et al. Automatic vessel lumen segmentation and stent strut detection in intravascular optical coherence tomography, J. Medical physics, 2012, 39(1): 503-513.

[5] Xu C, Schmitt J M, Carlier S G, et al. Characterization of atherosclerosis plaques by measuring both backscattering and attenuation coefficients in optical coherence tomography, J. Journal of biomedical optics, 2008, 13(3): 034003-034003-8.

[6] Ughi G J, Adriaenssens T, Sinnaeve P, et al. Automated tissue characterization of in vivo atherosclerotic plaques by intravascular optical coherence tomography images, J. Biomedical optics express, 2013, 4(7): 1014-1030.

[7] Wang Z, Kyono H, Bezerra H G, et al. Semiautomatic segmentation and quantification of calcified plaques in intracoronary optical coherence tomography images, J. Journal of biomedical optics, 2010, 15(6): 061711-061711-10. 